\title{
TELEMÁTICA EDUCACIONAL E ENSINO DE QUÍMICA: CONSIDERAÇÕES SOBRE UM CONSTRUTOR DE OBJETOS MOLECULARES
}

\author{
EDUCATIONAL TELEMATICS AND THE TEACHING \\ OF CHEMISTRY: CONSIDERATIONS ABOUT A \\ CONSTRUCTOR OF MOLECULAR OBJECTS
}

TÉLÉMATIQUE ÉDUCATIONNELLE ET L'ENSEIGNEMENT

DE LA CHIMIE : CONSIDÉRATIONS SUR UN

CONSTRUCTEUR D'OBJETS MOLÉCULAIRES

TELEMÁTICA EDUCACIONAL Y ENSEÑANZA DE QUÍMICA: CONSIDERACIONES ACERCA DE UN

CONSTRUCTOR DE OBJETOS MOLECULARES

\author{
Marcelo Giordan * \\ Jackson Góis ${ }^{* *}$
}

\section{RESUMO}

Neste artigo, tratamos de um aspecto específico da representação de partículas por meio de aplicativos computacionais e como essa forma relativamente recente de representação se insere na educação cientifica de alunos da escola básica. Inicialmente, abordamos alguns aspectos gerais da representação na química nos apoiando nas contribuiçôes da comunidade de pesquisa em ensino de química. Em seguida, tratamos da representação das partículas também resgatando alguns estudos na área de educação em ciências, para então apresentar as contribuiçôes dos estudos sobre o uso de aplicativos de visualização, com ênfase na distinção entre animaçōes e simulaçôes. Na segunda parte, descrevemos as funcionalidades de um aplicativo de construção de objetos moleculares que vem sendo desenvolvido no nosso laboratório e suas primeiras aplicaçôes nas salas de aula do ensino médio.

Palavras-chave: Representação estrutural. Simulação. Animação. Educação em ciências. Ensino de química.

* Bacharel em Química e Doutor em Ciências pela Universidade Estadual de Campinas (Unicamp, 1997). Pós-doutor no Centro de Linguagem e Comunicação da Faculdade de Educação da Open University (Inglaterra, 2003). Professor dos programas de Pós-graduação em Educação e em Ensino de Ciências da Universidade de São Paulo - USP (giordan@fe.usp.br).

** Bacharel e licenciado em Química, Mestre em Bioquímica pelo Instituto de Química da USP. Estudante do programa de Pós-graduação em Ensino de Ciências da mesma universidade (jacksong@fe.usp.br).

Agradecimentos: ao CNPq pela concessão de recursos (processo 403223/03-2) e à Pró-reitoria de Graduação da USP pela concessão de bolsa a Jackson Góis. 


\section{INTRODUÇÃO}

O ser humano tem se ocupado da matéria desde sua própria constituição enquanto espécie, na medida em que sua sobrevivência está assentada na interação com o mundo natural que lhe provê sustento e abrigo, mas que também o desafia com as intempéries e inimigos. Seja para caçar, plantar, lutar ou procriar, a manipulação dos materiais é uma ação prioritária para a espécie. Assim, não se pode compreender o percurso históricocultural do ser humano sem considerar o papel dos materiais nas açôes características da espécie, fundamentalmente na estruturação de atividades cognitivas superiores e na construção da base material para as interações de caráter social e técnico.

Da mesma forma, o controle sobre a produção e transformação de materiais tem sido determinado pela construção de um conhecimento sobre a constituição desses materiais. Se no passado o ser humano se concentrava em produzir e transformar os materiais tendo como referência apenas suas propriedades tangíveis, nos dias de hoje o controle sobre a manipulação da matéria ocorre no âmbito de sua constituição molecular. É fato que o acesso ao mundo das partículas constituintes da matéria está profundamente enraizado em formas de pensamento e em uma linguagem construídas nas interações realizadas no seio de comunidades com estatuto cultural e histórico próprios. Portanto, não se pode compreender a formação do pensamento e da linguagem das ciências que lidam com a transformação dos materiais sem considerar como ocorrem, não apenas as interações dos materiais, mas fundamentalmente como se realizam as açôes nessas comunidades.

Neste artigo, tratamos de um aspecto específico da representação de partículas por meio de aplicativos computacionais e como essa forma relativamente recente de representação se insere na educação científica de alunos da escola básica. Inicialmente, abordamos alguns aspectos gerais da representação na química nos apoiando nas contribuições da comunidade de pesquisa em ensino de química. Em seguida, tratamos da representação das partículas também resgatando alguns estudos na área de educação em ciências, para então apresentar as contribuições dos estudos sobre o uso de aplicativos de visualização, com ênfase na distinção entre animaçōes e simulações. Na segunda parte, descrevemos as funcionalidades de um aplicativo de construção de objetos moleculares que vem sendo desenvolvido no nosso laboratório e suas primeiras aplicações nas salas de aula do ensino médio.

\section{SOBRE AS FORMAS DE REPRESENTAR O CONHECIMENTO QUÍMICO}

Há um certo consenso em torno da idéia de o conhecimento químico ser construído pela combinação de três dimensões da realidade: macroscópica, microscópica e simbólica (JOHNSTONE, 1993; GABEL; BUNCE, 1994; GARNET et al., 1995; DORI et al., 1996; GABEL, 1998; BOWEN, 1998; ARDAC; AKAYGUN, 2004). Parte dos fenômenos e processos químicos são perceptíveis e observáveis através de informações sensoriais e medições que se concretizam em uma dimensão macroscópica. Dentro do paradigma atômico-molecular vigente, no qual a natureza particulada da matéria é a fundamentação teórica para interpretar esses fenômenos e processos, admite-se 
uma outra dimensão da realidade onde ocorrem fenômenos envolvendo o movimento e a interação de partículas. Em uma dimensão simbólica, substâncias, partículas e transformações são representadas por meio de símbolos, fórmulas e equações químicas, bem como expressões algébricas, tratando-se, portanto, de uma materialização semiótica da realidade.

Segundo Hoffman e Laszlo (1991), as representações simbólica e microscópica evoluíram de analogias fenomenológicas de experiências sensoriais no nível macroscópico, as quais permitem aos químicos terem uma linguagem comum para sua investigação conjunta, e são utilizadas para a comunicação entre profissionais da comunidade (KOZMA; CHIN; RUSSEL; MARX, 2000). Ainda de acordo com Hoffman e Laszlo, as representações químicas são metáforas, modelos ou construtos teóricos da interpretação química da natureza e da realidade, com o que está de acordo Nye (1993), que também sugere serem essas características determinantes da formação de um pensamento que diferencia a química de outras ciências.

Professores e pesquisadores do ensino de química têm realizado estudos sobre como promover o entendimento conceitual em estudantes do ensino médio (WU; KRAJCIK; SOLOWAY, 2001) e superior (KOZMA; RUSSEL, 1997) através do desenvolvimento da habilidade de representação das três dimensões do conhecimento químico. Nesses estudos percebeu-se que os estudantes parecem não dominar as construções simbólicas da química, tratando equações químicas como entes matemáticos, ao invés de pensar nas mesmas como representações de processos dinâmicos e interativos. Outras pesquisas apontam para o fato de estudantes poderem elaborar a resposta correta para problemas em química tendo apenas um entendimento conceitual limitado (SAWYER, 1990; SMITH; METZ, 1996), sem que tenham se apropriado, por exemplo, da simbologia associada. É neste sentido que se tem defendido a resolução satisfatória de problemas desafiando o estudante a se apropriar de dispositivos de pensamento da química, o que é observado em situações que lhes permitam correlacionar o fenômeno em sua dimensão simbólica com a representação simbólica e microscópica.

Estudos também apontam para o fato de estudantes de ensino médio e superior apresentarem dificuldade para compreender fenômenos e transformações químicas em termos do modelo de partículas atualmente aceito (GARNET et al., 1995). Alguns autores têm interpretado essas dificuldades de aprendizagem como oriundas da natureza particulada, abstrata e não observável da química, e da necessidade de rápida transferência entre os três níveis de representação (JOHNSTONE, 1991; GABEL et al., 1992).

Existe uma dificuldade maior por parte dos estudantes na compreensão do nível microscópico e na representação do nível simbólico, pelo fato de as mesmas serem invisíveis e abstratas. Desta forma, devido ao fato de o pensamento dos estudantes se basear em informaçōes sensoriais, estes têm a tendência em permanecer no nível macroscópico em suas explicações sobre os fenômenos e propriedades de substâncias (BEN-ZVI; EYLON; SILBERSTEIN, 1987, 1988; GRIFFTHS; PRESTON, 1992).

Professores, pesquisadores e profissionais da química operam apropriadamente entre as dimensōes do conhecimento, enquanto estudantes têm dificuldade em estabelecer ligaçōes entre esses níveis. Desta forma, parece bastante provável que a utilização de modelos, analogias e gráficos computacionais em situações estruturadas de ensino seja 
produtiva para os estudantes se apropriarem das formas de pensamento químico, conforme alguns estudos têm sugerido. É necessário, portanto, considerar tanto a fundamentação epistemológica da química nas especificidades do seu paradigma atômicomolecular, quanto a organização das atividades de ensino, quando nos propomos a introduzir um meio mediacional estranho à sala de aula e à própria concepção de ensino de química predominante nas escolas de ensino médio.

\section{USO DE MODELOS MOLECULARES NO ENSINO DE QUÍMICA}

A representação dos níveis de conhecimento através de múltiplos meios tem sido eficaz no ensino de química, através da utilização de diferentes sistemas de símbolos para representar informações em diferentes formas (KOZMA, 1991). As características superficiais de cada sistema de símbolos podem melhor representar certas características da informação (KOZMA; RUSSEL, 1997).

Pesquisadores têm sugerido diferentes abordagens instrucionais como apoio ao ensino de química, como adaptação de estratégias de ensino baseado no modelo de mudança conceitual (KRAJCIK, 1991), integração de atividades de laboratório na aula em classe (JOHNSTONE; LETTON, 1990), uso de modelos concretos (COPOLO; HOUNSHELL, 1995) e uso de tecnologias como ferramentas de aprendizado (BARNEA; DORI, 1999; KOZMA; RUSSEL; JONES; MARX; DAVIS, 1996; WU; KRAJCIK; SOLOWAY, 2001). O uso de modelos concretos juntamente com tecnologias como ferramentas de aprendizado parece promissor (WU; KRAJCIK; SOLOWAY, 2001).

Estudos têm indicado bons resultados de aprendizagem quando da utilização de objetos moleculares concretos como forma de visualização do modelo de partículas e das transformações químicas associadas (COPOLO; HOUNSHELL, 1995; GABEL; SHERWOOD, 1980; TALLEY, 1973). Alguns autores têm observado um efeito cumulativo de longo prazo na compreensão dos estudantes sobre os fenômenos quando são submetidos à manipulação desses objetos moleculares concretos (GABEL; SHERWOOD, 1980). Esse tipo de visualização é apontado com um dos mais utilizados na atualidade, pois simplifica, ilustra e permite a exploração da estrutura e do processo químico associado. No entanto, esses objetos são rígidos e geralmente em quantidade limitada, o que restringe seu uso à representação de moléculas pequenas (BARNEA; DORI, 1999).

Uma das funcionalidades dos aplicativos computacionais é veicular animações em nível molecular do fenômeno químico, o que não é possível através de outros meios de representação. A utilização dessa tecnologia como ferramenta de ensino permite a visualização de animações dinâmicas projetadas tridimensionalmente, o que tem auxiliado estudantes a representar simbolicamente os processos químicos e, portanto, a interpretar a fenomenologia nas dimensōes macroscópica e microscópica (WILLIAMSON; ABRAHAM, 1995). Este suporte ao aprendizado provido pelas tecnologias computacionais tem sido considerado como um atributo específico e particularmente útil desses meios para representar as três dimensões do pensamento químico, pois elas têm a qualidade de dispor a informação em sistemas simbólicos diferentes, mas coordenados 
(KOZMA, 1991). Representações múltiplas ligadas entre si permitem ao estudante visualizar interações entre moléculas e entender os conceitos químicos relacionados (KOZMA et al., 1996).

Um problema que os estudantes freqüentemente enfrentam é a realização de tarefas que exigem habilidades de visualização tridimensional, bem como a visualização tridimensional de moléculas que são representadas bidimensionalmente em livros (BARNEA; DORI, 1999). Há evidências de que esse tipo de representação, especialmente quando animada e dinâmica, pode aprimorar a visualização tridimensional dos estudantes (SEDDON; SHUBBER, 1985; SEDDON; MOORE, 1986; TUCKEY et al., 1991). Estudos mostraram que a construção de conceitos está estreitamente relacionada ao formato visual com que os estudantes tiveram contato durante seu aprendizado (CLARK; PAIVIO, 1991; PAIVIO, 1986). É neste sentido que alguns autores têm defendido a integração entre gráficos computacionais e representação tridimensional como uma forma efetiva de aprimorar a habilidade de visualização no ensino de ciências (KISER, 1990; RODRIGUEZ, 1990; WILEY, 1990; BEZZI, 1991; BARNEA; DORI, 1996).

A utilização de objetos moleculares virtuais gerados por computadores, além de permitir a disposição de múltiplas representaçōes coordenadas e tridimensionalmente projetadas, também favorece variados tipos de manipulação desses objetos, como translação, rotação, aumento ou redução de tamanho. Uma outra vantagem da visualização computacional é a possibilidade de se representar moléculas de, virtualmente, qualquer tamanho, dependendo apenas da capacidade de processamento do computador. É neste sentido que o desenvolvimento de aplicativos computacionais para atividades de ensino se apresenta como uma alternativa potencialmente transformadora das práticas escolares e da construção de conhecimento entre estudantes, desde que seja considerada a correlação das três dimensões do conhecimento químico na organização das atividades e se investiguem os ditames das ações mediadas pelos aplicativos, que são fundamentalmente diferentes daquelas realizadas em situações de ensino ancoradas na experimentação ou qualquer outra forma de acesso à fenomenologia, isso porque o estatuto do fenômeno se altera radicalmente quando é transposto da bancada para a tela do computador (GIORDAN, 1999).

\section{SIMULAÇÃO, ANIMAÇÃO EVISUALIZAÇÃO DE OBJETOS MOLECULARES PELO COMPUTADOR}

Consideremos algumas especificidades da representação de objetos moleculares no computador, tendo em vista duas formas distintas de visualizá-los: a animação e a simulação.

Animações computacionais são geradas a partir de aplicativos gerais de edição gráfica, sem necessariamente incluir valores empíricos de propriedades das substâncias ou das transformações obtidos em pesquisa cientifica, e intencionam enfatizar determinadas características superficiais macroscópicas ou microscópicas sem obedecer escalas de tempo ou tamanho. Já as simulações computacionais são geradas a partir de aplicativos específicos para estudo de propriedades de substâncias e transformações químicas, e estão 
intimamente relacionados ao ambiente de pesquisa cientifica. Para realizar essas simulaçōes são utilizados valores teóricos ou empíricos de propriedades químicas, como ângulos e distancias de ligações, e as escalas de tempo e tamanho são parametrizadas em equações matemáticas que satisfazem as leis físicas que descrevem os fenômenos.

Em qualquer um dos casos, o objeto molecular é uma representação imagética da entidade molecular e pode ser concebido como uma metáfora do que supomos ocorrer na dimensão microscópica da matéria, não sendo, pois, um retrato da realidade. Chamamos de objetos moleculares as representações das partículas microscópicas, cujo meio de veiculação pode variar desde o papel, passando pelos conjuntos plásticos, isopor e madeira, chegando à tela do computador ou à projeção holográfica (GIORDAN, 2005). Varia-se o meio e também as formas de representação, nesse caso com o objetivo de destacar uma ou outra propriedade da molécula. Portanto, o objeto molecular é uma entidade iconográfica que serve tanto para propósitos de indexação e referência, como para mimetizar determinada propriedade molecular, tendo esta a possibilidade de ser simulada por meio de um sistema de equações quando o objeto é veiculado pelo computador.

Pelo fato de as simulações levarem em consideração as propriedades do sistema em estudo, as mesmas podem ser visualizadas como objetos moleculares virtuais dinâmicos, uma vez que é possível simular a variação de propriedades como distância e ângulos de ligação. A partir dessas simulações podem ser gerados filmes simulando o movimento conjunto dos átomos nas moléculas e em sistemas moleculares.

Uma decorrência importante da manipulação de diversas formas de representação é a possibilidade de criar um efeito vinculante entre a variável, de natureza teórica, e a forma de representação da propriedade, de natureza imagética. A visualização de objetos moleculares mediada pelo computador parece, portanto, promover a vinculação entre a simulação de uma propriedade da molécula e sua representação em um mesmo meio. Essa é uma situação de alto valor didático, capaz de mobilizar as ações dos alunos na manipulação do objeto, na elaboração discursiva e também na elaboração de significado, conforme temos discutido em outro estudo (GIORDAN, 2005).

Apesar de existirem diversas ferramentas de visualização e construção de objetos moleculares virtuais específicas para pesquisadores, como, por exemplo, aplicativos de mecânica e dinâmica molecular, a utilização delas por alunos do ensino médio ou mesmo por alunos iniciantes no ensino superior em química é dificultada em razão da profundidade do conhecimento envolvido nos cálculos e no controle de variáveis. Em alguns casos, os estudantes precisam fornecer valores para variáveis, como constantes de força de ligações químicas, conhecer detalhes do processo de minimização de energia ou ainda detalhes sobre a organização das informações de saída fornecidas pelos aplicativos. Assim, para que os estudantes tenham acesso às aplicações de visualização molecular baseadas em simulações, é necessário desenvolver ferramentas que simplifiquem a transferência de dados entre as interfaces de entrada e saída e simultaneamente possibilitem o controle sobre variáveis que afetam a visualização.

Alguns grupos têm se dedicado a desenvolver interfaces para prover os estudantes com ferramentas computacionais que permitam a visualização de objetos moleculares virtuais. Russel e Kozma (1994) utilizaram um software protótipo de ambiente (4M:Chem) 
que incorpora simultânea e sincronizadamente representações múltiplas de fenômenos químicos. A utilização do módulo com animações sobre equilíbrio gasoso indicou um aumento do conhecimento dos estudantes sobre as características de sistemas no equilíbrio e o efeito da temperatura nesses sistemas. Avaliações sobre a utilização desse sistema indicaram uma diminuição de declaraçōes errôneas sobre conceitos químicos.

Wu, Krajcik e Soloway (2001) utilizaram uma versão simplificada de ferramentas profissionais centradas no estudante (eChem), para auxiliar estudantes de ensino médio a construir modelos, visualizar múltiplos modelos tridimensionais e comparar representações macroscópicas. Ao invés de oferecer modelos construídos prontos, o software ofereceu a oportunidade de os estudantes construírem seus próprios modelos e externalizarem suas representaçōes. Nesse software, cada molécula, para ser visualizada, deve ser construída átomo por átomo e ligação por ligação, sendo que o tipo de ligação deve ser especificado.

Ardac e Akaygun (2004) desenvolveram uma ferramenta de visualização química (Chemical Change) sobre transformações químicas, a qual enfatiza as representaçôes macroscópicas, simbólicas e microscópicas. Através do uso de animações, o software oferece os três tipos de representação simultaneamente aos estudantes. Além disso, o software propõe situações nas quais é necessária a interação com material de suporte instrucional apostilado, de forma que são requeridos dos estudantes desenhos e respostas escritas como resultado final.

Não temos conhecimento até o presente momento de aplicativos de visualização molecular que permitam aos estudantes produzir suas próprias representações de sistemas moleculares por meio de interfaces simplificadas que não exijam conhecimento aprofundado das teorias de modelagem molecular. Diante dessa lacuna, que provavelmente tem impedido a utilização de computadores em situações de ensino-aprendizagem de química, temos desenvolvido um projeto que, em sua fase de produção de aplicativos, consta de duas fases: a criação de uma interface de comunicação do usuário com os aplicativos de simulação para construção de objetos moleculares e o desenvolvimento da interface de veiculação do objeto molecular na tela do computador. A seguir, passamos a descrever as funcionalidades do aplicativo de construção de objetos moleculares.

\section{CONSTRUTOR}

Construtor é uma ferramenta de criação de objetos moleculares virtuais que utiliza o protocolo de transferência de hipertexto (http) para realizar a comunicação do computador cliente com o servidor. Através dessa interface, o estudante pode construir animações de modelos moleculares bidimensionais, e também pode construir e visualizar, por meio de um plug-in, simulaçôes desses modelos projetadas tridimensionalmente a partir da fórmula estrutural condensada. As animaçôes e as simulações estão dispostas em uma mesma tela, para que o estudante tenha oportunidade de comparar suas próprias representações com representaçôes associadas ao modelo cientificamente aceito. As simulações são feitas utilizando o programa de modelagem molecular Tinker (PONDER; RICHARDS, 1987), e visualizadas com o plug-in Chime, ambas aplicações de domínio público e amplamente empregadas no meio científico. 
O ambiente de animação gráfica foi escrito com o auxilio do aplicativo Flash®, da Macromedia, que é um aplicativo de uso geral na construção de animações gráficas. Nesse aplicativo, desenhos bidimensionais podem ser criados a partir de figuras geométricas simples. As ferramentas oferecidas fornecem opçōes onde os desenhos criados podem simular a sensação visual de tridimensionalidade. Os desenhos podem ser criados em uma quantidade variável de quadros com posicionamentos diferentes, de forma que a sobreposição dos quadros cria a sensação visual de movimentação do desenho criado. A possibilidade de criação de desenhos diferentes em camadas separadas, como parte de uma mesma animação, favorece um ambiente de edição organizado e planejado.

O programa em execução no computador servidor, o qual interage com o ambiente de simulação tridimensional do usuário, e fornece os arquivos apropriados para visualização, foi escrito em linguagem C padrão ANSI para ser executado em servidor com ambiente GNU/Linux, da distribuição RedHat 9.0. Essa parte do ambiente oferece uma caixa de entrada para o usuário enviar ao computador servidor, através de protocolo de comunicação via hipertexto da Internet, uma seqüência de letras e números correspondentes à fórmula estrutural condensada de uma molécula orgânica, como por exemplo $\mathrm{CH}_{3} \mathrm{CH}_{2} \mathrm{CH}_{3}, \mathrm{CH}_{3} \mathrm{CH}_{2} \mathrm{CHO}, \mathrm{CH}_{3} \mathrm{CH}_{2} \mathrm{COCH}_{3}$ ou $\mathrm{CH}_{3} \mathrm{OCH}_{2} \mathrm{CH}_{3},{ }^{1}$ inclusive com cadeia ramificada, insaturada e cíclica. Até o presente momento o programa Construtor reconhece seqüências correspondentes a hidrocarbonetos, compostos halogenados, álcoois, aldeídos, cetonas e éteres. O programa Construtor, em execução no servidor, recebe a seqüência de letras e números, e devolve como resposta um arquivo correspondente à fórmula tridimensional geometricamente otimizada da molécula em questão, ao navegador do usuário. $\mathrm{O}$ tempo necessário para a construção do arquivo no servidor depende da quantidade de átomos desejada. Para moléculas com até 20 átomos o tempo de processamento não ultrapassa 1 segundo em uma CPU com processador Celeron 333 $\mathrm{MHz}$. O tempo de resposta naturalmente irá depender da velocidade de conexão do usuário com a internet. $\mathrm{O}$ usuário pode visualizar o arquivo automaticamente em seu navegador se instalar em seu computador um plug-in de visualização amplamente conhecido e utilizado no meio acadêmico. ${ }^{2}$ A figura 1 apresenta o organograma de funcionamento do Construtor: 
Figura I: Organograma de funcionamento do aplicativo Construtor.

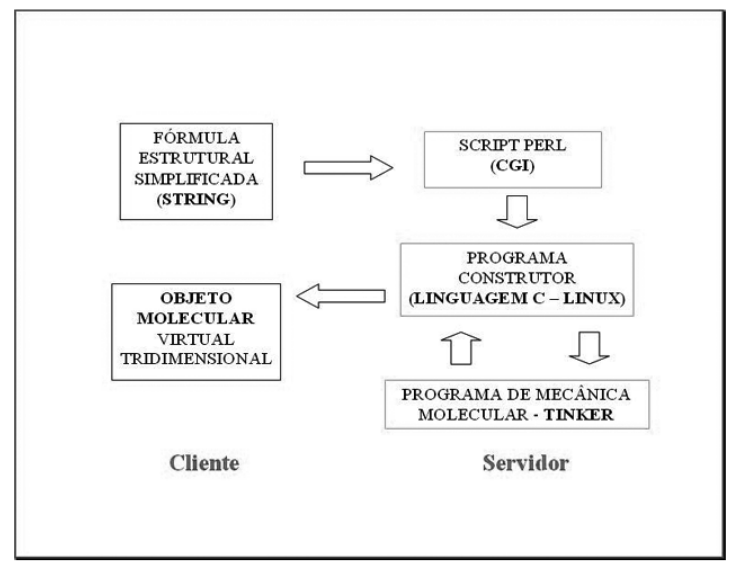

Passaremos a descrever o funcionamento do programa Construtor, bem como sua interface com o recebimento e envio de arquivos pela internet. A página tipo hipertexto, de onde Construtor pode ser acessado, disponibiliza um campo onde o usuário escreve a seqüência de letras e números correspondente à fórmula estrutural condensada de uma molécula orgânica. Próximo a esse campo existe um botão que, ao ser acionado pelo usuário, envia a seqüência escrita ao computador servidor. A página hipertexto, através do método POST, envia a seqüência de letras e números para um script tipo CGI, escrito em linguagem Perl, presente no servidor em diretório apropriado. A receber esta seqüência, o script é executado no servidor e se encarrega de processar comandos e executar programas, e finalmente devolver o arquivo final ao navegador do usuário como resposta. Inicialmente o script processa a seqüência de letras e números, para que essa seqüência seja formatada apropriadamente, de forma a ser reconhecida pelos próximos comandos e programas. A seqüência formatada é enviada pelo script para um programa escrito em linguagem C (KERNIGHAN, 1988) padrão ANSI chamado Construtor. O Programa Construtor, a partir da seqüência recebida, constrói um arquivo em coordenadas tridimensionais e também a matriz de conectividade apropriada, que indica quais átomos estão ligados entre si. $\mathrm{O}$ arquivo correspondente é gerado em formato próprio para utilização do pacote de programas de mecânica e dinâmica molecular Tinker, livre para uso acadêmico e disponível na internet. ${ }^{3} \mathrm{O}$ programa Construtor, após gerar o arquivo de coordenadas tridimensionais correspondente à seqüência recebida, submete esse arquivo à otimização de geometria por um método de minimização de energia, utilizando o programa Minimize do pacote Tinker. Os parâmetros adequados para serem utilizados automaticamente na otimização de geometria foram selecionados de forma a oferecer uma geometria apropriadamente otimizada, mas também de forma a não demandar demasiado tempo de processamento no servidor. Este último arquivo é devolvido pelo script ao navegador do usuário, também através de protocolo de comunicação via hipertexto, finalizando o trabalho realizado pelo computador servidor. 


\section{INTERFACES COM O USUÁRIO}

\section{Animação}

A interface de animação do programa Construtor é capaz de gerar animações estáticas bidimensionais, que não desenvolvem movimentação após serem construídas. O programa não apresenta a opção de movimentação dos átomos e moléculas, porque o mesmo é utilizado pelos alunos em um estagio de conhecimento inicial, logo após a manipulação de objetos moleculares concretos e registro de estruturas moleculares em papel. Na figura 2, apresenta-se uma tela do aplicativo Construtor e também da interface de visualização produzida pelo plug-in Chime.

\section{Figura 2: Interface gráfica do aplicativo Construtor.}

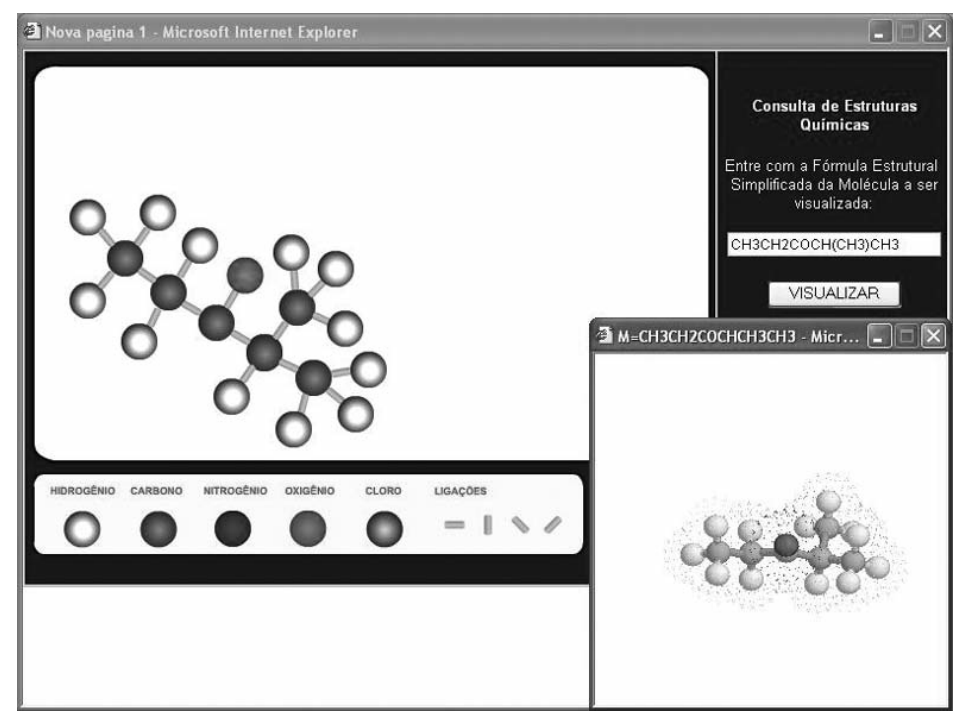

No ambiente de animação gráfica, os estudantes dispõem de uma barra de ferramentas na parte inferior da tela. Nesta barra de ferramentas estão disponibilizadas figuras geométricas bidimensionais que funcionam como objetos virtuais, manipuláveis pelo usuário em quantidade e posicionamento. A forma geométrica desses objetos mimetiza o formato dos objetos moleculares concretos do tipo bola-vareta, de forma que existem 'bolas' e 'varetas' virtuais em quantidade ilimitada, bem como um espaço bidimensional à disposição dos estudantes, onde os mesmos podem ser manipulados. Escolhemos oferecer inicialmente aos estudantes uma área de trabalho bidimensional, bem como objetos moleculares bidimensionais, para evitar obstáculos para aprendizagem, pois a manipulação de objetos em ambiente com projeção tridimensional requer o manuseio 
de uma quantidade maior de comandos, bem como comandos mais sofisticados, como, por exemplo, aqueles que produzem textura, sombra e profundidade nos objetos.

As bolas virtuais, que simbolizam os átomos, são apresentadas em diferentes colorações no padrão CPK, de modo a oferecer ao estudante diferentes tipos atômicos. Escolhemos esse padrão de coloração pelo fato de ele ser utilizado por grande parte dos sistemas de visualização empregados em livros e aplicativos. Os modelos concretos tipo bola-vareta utilizados em situaçōes de sala de aula foram fabricados com base nesse padrão de cores, o que favorece a correlação entre os objetos moleculares concretos e virtuais.

Os objetos moleculares que representam as ligaçōes químicas virtuais são bastōes de tamanhos idênticos, porém estão dispostos espacialmente em quatro posições diferentes. Qualquer uma dessas ligaçóes químicas pode ser rotacionada em $360^{\circ}$, de forma a tomar o posicionamento apropriado, de acordo com o ponto de vista dos estudantes. Optamos em oferecer esses objetos em quatro posicionamentos diferentes para permitir ao estudante selecionar aquele que mais se aproxima da posição que ele necessita para construir suas representaçōes. Também escolhemos restringir a um único tamanho esses objetos, porque o objetivo da atividade durante a utilização desse ambiente está focado na visualização das posições relativas dos átomos, bem como da ordem de ligação.

A manipulação desses objetos moleculares virtuais, através de animações gráficas, retém a simplicidade e a conveniência da manipulação dos objetos moleculares concretos já utilizados, com a vantagem da possibilidade de construção de moléculas de qualquer tamanho. Nessa interface os estudantes podem mover livremente cada um dos objetos que simbolizam os átomos e as ligaçôes químicas pela tela do computador. Apesar disto, como os estudantes já manipularam os objetos moleculares concretos, eles têm a tendência de agrupar os objetos bidimensionais que simbolizam os átomos e ligaçōes químicas numa disposição próxima àquela que mimetiza o posicionamento relativo tridimensional.

\section{Simulação}

A construção dos objetos moleculares virtuais através do ambiente de simulação é operacionalmente simples, pois requer que o estudante apenas digite uma seqüência de letras e números correspondentes à fórmula estrutural condensada de uma molécula orgânica, e escolha uma forma de representação entre as disponíveis (bola-vareta, espaço preenchido, traço, esqueleto e nuvem eletrônica). Em seguida, o estudante deve pressionar o botão esquerdo do mouse sobre a opção "construir", oferecida logo abaixo do campo onde digita a fórmula estrutural condensada da molécula em questão. Essa sequêencia de letras e números é enviada ao nosso computador servidor, o qual gera um arquivo contendo as coordenadas tridimensionais geometricamente otimizadas da molécula em questão. O computador servidor devolve esse arquivo ao navegador do usuário, que pode visualizar o objeto molecular caso o plug-in de visualização.

Apesar de ser operacionalmente simples, a utilização do ambiente de simulação requer operaçõos mentais mais sofisticadas do que o ambiente de animação gráfica, uma vez que o estudante deve desenvolver um domínio da representação simbólica, pelo fato de a interface de construção demandar sequiências de letras e números corretas, ou seja, que correspondam a uma molécula dentro dos padrões atualmente aceitos. Ao utilizar esse ambiente, o estudante é desafiado a visualizar moléculas a partir de seus nomes no 
padrão IUPAC, ${ }^{4}$ de forma que tem a oportunidade de exercitar a representação da fórmula estrutural a partir do nome, e também tem a oportunidade de verificar se sua representação corresponde ao modelo aceito.

Ao manipular objetos moleculares virtuais tridimensionais, o estudante pode explorar o objeto molecular sob diversas perspectivas com o auxílio do mouse, mimetizando a forma como se manipulam os objetos moleculares concretos. Isto faz com que os estudantes criem uma correlação entre o mundo concreto e o virtual, o que pode tornar mais estreita a correlação entre suas imagens mentais e as representações simbólicas e objetos moleculares concretos e virtuais. Além de visualizar a fórmula estrutural em três dimensões, é possível medir os ângulos e distâncias de ligação no objeto molecular virtual. Com isso, o estudante pode comparar, por exemplo, os comprimentos de ligação das ligações simples e insaturadas, o que não é possível em um ambiente de animação gráfica ou com os objetos concretos. Além disso, o estudante pode comparar os diferentes volumes atômicos dos átomos e grupos atômicos presentes na molécula, o que também não é possível a partir de outras formas de representação.

Assim, ambientes que possibilitem o contato do estudante com simulações favorecem o aprofundamento de conceitos que podem ser introduzidos a partir de representações menos sofisticadas, como o de ligações químicas.

\section{APLICAÇÕES INICIAIS E PERSPECTIVAS FUTURAS}

Foi realizado um teste simples de usabilidade do programa Construtor com 32 estudantes do terceiro ano do Ensino Médio de uma escola pública do Estado de São Paulo, visando coletar informaçôes para aprimorar a versão atual. Os estudantes tiveram acesso ao programa Construtor utilizando o Tutorial de Química Orgânica (TQO) como material instrucional de apoio, o qual foi utilizado como atividade orientadora das aulas. Além das respostas por escrito, foi solicitada aos estudantes a realização de atividades de construção, manipulação e visualização de objetos moleculares virtuais e concretos. Os objetos moleculares concretos foram colocados à disposição na forma de modelos comerciais tipo bola e vareta, enquanto os objetos moleculares virtuais foram exibidos aos estudantes como exemplos, ou construídos a partir das interfaces de animação e simulação.

Os estudantes foram organizados em 16 duplas, com o objetivo de incentivar a verbalização e socialização de idéias. Durante as aulas com o programa Construtor, os estudantes tiveram acompanhamento do professor, que foi consultado quando surgiram dúvidas sobre o enunciado das questôes e atividades propostas, ou quando discordavam entre si da resposta a ser dada.

Nessas aulas, realizadas em uma sala com 18 computadores, os estudantes mostraram atitudes positivas, tanto em relação ao material, quanto às atividades que eram solicitadas, mesmo aqueles que não participavam ativamente de atividades similares, sem o uso de computadores, na sala de aula. As respostas dos alunos às atividades e questôes propostas trouxeram indícios da apropriação da simbologia adequada ao modelo de partículas, quando interpretaram fenômenos e propriedades macroscópicas. 
De modo geral, observou-se boa desenvoltura dos estudantes com as interfaces, não tendo sido registrada qualquer dificuldade operacional das interfaces de animação e simulação. Testes de usabilidade mais refinados estão sendo planejados a partir dos dados de registro da tela em que os estudantes trabalham, concomitantemente ao registro audiovisual dos mesmos. Com o desenvolvimento de um sistema de ajuda, realizaremos testes mais específicos.

Outra fase do projeto centra-se no desenvolvimento de uma interface para visualização baseada na plataforma Java 2 e usando a API JOGL (Java bindings for OpenGL), de modo que, além das funcionalidades já disponíveis no Chimieß, sejam incorporados movimentos que mimetizem o movimento de vibração atômico. Estudamos a possibilidade de converter representações bidimensionais de objetos moleculares, desenhadas na interface de animação, em arquivo de entrada para a interface de simulação, oferecendo assim uma opção de criação de objetos que prescinda da entrada de dados a partir da fórmula estrutural condensada. Essa interface de conversão direta poderá ser utilizada por estudantes de ensino superior que já têm a simbologia química em um grau elevado de apropriação.

O desenvolvimento de interfaces computacionais para visualização de objetos moleculares reacende uma discussão tão antiga quanto atual: como o ser humano age com a matéria para transformar o mundo. Um dos temas contemporâneos mais instigantes, ao lado dos meios digitais e da internet, é a manipulação da matéria em escala nanoscópica. Criar dispositivos em escala molecular é a nova fronteira de manipulação da matéria pela espécie. Produzir substâncias de interesse farmacológico a partir de usinas enzimáticas selecionadas também faz parte da agenda da indústria de química fina. A nanotecnologia e a biologia molecular são fronteiras do conhecimento que conhecerão rápida expansão neste século que se inicia.

A inserção da população nesse debate é uma prioridade do letramento científico que não pode ser excluído da educação básica. Hoje, as implicações dessas formas de manipular a matéria atingem dimensões éticas que exigem de nós a formação de um juízo que extravase os limites da ciência, mas não descarte a compreensão de como se constrói o conhecimento também em sua dimensão material. Levar computadores para escolas é mais do que atender as demandas do mercado de trabalho, significa cultivar formas de pensamento contemporâneas, que estão enraizadas no desenvolvimento da espécie, pelo menos na sua ânsia por dominar a matéria. 


\section{Notas}

1. A interface ainda não se encontra adaptada para trabalhar com sub-índices.

2. Disponível em http://www.mdl.com.

3. Disponível em http://dasher.wustl.edu/tinker.

4. Refere-se a International Union of Pure and Applied Chemistry.

\section{Referências}

ARDAC, D.; AKAYGUN, S. Effectiveness of multimedia-based instruction that emphasizes molecular representations on students' understanding of chemical change. Journal of Research in Science Teaching, 2004, v. 41, n. 4, p. 317.

BARNEA, N.; DORI, Y. J. High School chemistry students' performance and gender differences in a computerized molecular modeling learning environment. Journal of Science Education and Technology, 1999, v. 8, n. 4, p. 257-271.

BARNEA, N.; DORI, Y. J. Computerized molecular modeling as a tool to improve chemistry teaching. Journal of Chemical Information and Computer Sciences, 1996, v. 36, p. 629-636.

BEN-ZVI, R.; EYLON, B.; SILBERSTEIN, J. Students' visualization of a chemical reaction. Education in Chemistry, July 1987, p. 117-120.

BEN-ZVI, R.; EYLON, B.; SILBERSTEIN, J. Theories, principles and laws. Education in Chemistry, May 1988, p. 89-92.

BEZZI, A. A Macintosh program for improving three dimensional thinking. Journal of Geological Education, 1991, n. 39, p. 284-288.

BOWEN, C.W. Item design considerations for computer-based testing of student Learning in chemistry. Journal of Chemical Education, 1998, n. 75, p. 1.172-1.175.

CLARK, J. M.; PAIVIO, A. Dual coding theory and education. Educational Psychology Review, 1991, n. 3, p. 149-210.

COPOLO, C.F.; HOUNSHELL, P.B. Using three-dimensional models to teach molecular structures in high school chemistry. Journal of Science Educacion and Technology, 1995, v. 4, n. 4, p. 295-305. DORI, Y.J.; GABEL, D.; BARNEA, N.; HAMEIRI, M. Using novel Technologies to enhance chemistry understanding at the phenomena, molecular and symbolic levels. Proceeding of the Second Jerusalem International Science and Technology Education Conference, Jerusalem, Israel, S240a. 1996.

GABEL, D.L. The complexity of chemistry and implications for teaching. In: FRASER, B.J.; TOBIN, K.G. (Eds.). International Handbook of Science Education, Kluwer Academic Publishers, London, 1998, p. 233-248.

GABEL, D.L.; BUNCE, D. M. Research on problem solving: chemistry. In: GABEL, D. L. (Ed.). Handbook of research on Science Teaching and Learning. New York: Macmillan, 1994, p. 301-326. GABEL, D.L.; BRINER, D.; HAINES, D. Modeling with magnets - A unified approach to chemistry problem solving. The Science Teacher, March 1992, p. 58-63.

GABEL, D.; SHERWOOD, R. The effect of student manipulation of molecular models on chemistry achievement according to Piagetian level. Journal of Science Teaching, 1980, v. 17, n. 1, p. 75-81. GARNET, P.J.; GARNET. P.J.; HACKING, M. W. Students' alternative conceptions in chemistry: A review of research and implications for teaching and learning. Studies in Science Education, 1995, n. 25, p. 69-95. 
GRIFFITHS, A.K.; PRESTON, K.R. Grade-12 students' misconceptions relating to fundamental characteristics at atoms and molecules. Journal of Research in Science Teaching, 1992, v. 29, n. 6, p. 611-628.

GIORDAN, Marcelo. O papel da experimentação no ensino de ciências. Química Nova na Escola, 1999, n. 10, p. 43-49.

GIORDAN, Marcelo. O computador no ensino de ciências: breve revisão crítica acerca de algumas formas de uso. Ciência e Educação. No prelo. 2005.

HOFFMANN, R.; LAZLO, R. Representation in chemistry. Angewandte Chemie, 1991, v. 30, p. 1-16. JONSTONE, A.H. The Development of chemistry teaching: A changing response to changing demand. Journal of Chemical Education, 1993, n. 70, p. 701-704.

JONSTONE, A.H. Why is science difficult to learn? Things are seldom what they seem. Journal of Computerized Assisted Learning, 1991, n. 7, p. 75-83.

JONSTONE, A.H.; LETTON, K.M. Investigating undergraduate lab work. Education in Chemistry, 1990, n. 28, p. 81-83.

KERNIGHAN, B.; RITCHIE, D. C, a linguagem de programação: padrão ANSI, Rio de Janeiro: Campus, 1989.

KISER, L. Interaction of spatial visualization with computer enhanced and traditional presentations of linear absolute value inequalities. Journal of Computers in Mathematics and Science Teaching, 1990 , v. 10 , p. $85-96$.

KOZMA, R.B. Learning with media. Review of Educational Research, 1991, n. 61, p. 179-211.

KOZMA, R.B.; CHIN, E.; RUSSELL, J.; MARX, N. The roles of presentations and tools in the chemistry laboratory and their implications for chemistry instruction. Journal of the Learning Sciences, 2000, v. 9, n. 2, p. 105-143.

KOZMA, R.B.; RUSSELL, J. Multimedia and understanding: Expert and novice responses to different representations of chemical phenomena. Journal of Research in Science Teaching, 1997, n. 34, p. 949-968.

KOZMA, R.B.; RUSSELL, J.; JONES, T.; MARX, N.; DAVIS, J. The use of multiple, linked representations to facilitate science understanding. In: VOSNIADOU, R.G.S.; DECORTE, E.; MANDEL, H. (Eds.). International perspective on the psychological foundations of technology-based learning environments (p. 41-60). Hillsdale, NJ: Erlbaum, 1996.

KRAJCIK, J.S. Developing students' understanding of chemical concepts. In: GLYNN, S.M.; YEANY, R.H.; BRITTON, B.K. (Eds.). The psychology of learning science: international perspective on the psychological foundations of technology-based learning environments (p. 117-145). Hillsdale, NJ: Erlbaum, 1991.

NYE, M.J. From chemical philosophy to theoretical chemistry. Berkeley, CA: University of California Press, 1993.

PAIVIO, A. Mental representations: a dual-coding approach. New York: Oxford University Press, 1986. PONDER, J.W.; RICHARDS, F.M. An efficient newton-like method for molecular mechanics energy minimization of large molecules.Journal of Computational Chemistry, 1987, n. 8, p. 1.016-1.024. RODRIGUEZ, W. E. A dual approach to engineering design visualization. Engineering Design Graphics Journal, 1990, v. 54, n. 3, p. 36-43.

RUSSEL, J.; KOZMA, R. 4M:Chem-multimedia and mental models in chemistry. Journal of Chemical Education, 1994, n. 71, p. 669-670.

SAWYER, B.A. Concept learning versus problem solving: revisited. Journal of Chemical Education, 1990 , n. 67, p. 253-254. 
SEDDON, G.M.; MOORE, R.G. An unexpected effect in the use of models for teaching the visualization of rotation in molecular structures. European Journal of Science Education, 1986, n. 8, p. 79-86.

SEDDON, G. M.; SHUBBER, K. E. The effects of color in teaching the visualization of rotation in diagrams of three dimensional structures. British Educational Research Journal, 1985, n. 11, p. 227-239.

SMIT, K.; METZ, P. Evaluating student understanding of solution chemistry through microscopic representations. Journal of Chemical Education, 1996, v. 73, p. 233-235.

TALLEY, L. The use of three-dimensional visualization as a moderator in the higher cognitive learning of concepts in college level chemistry. Journal of Research in Science Teaching, 1973, v. 12, p. 41-43.

TUCKEY, H.; SELVARATNAM, M.; BRADLEY, J. Identification and rectification of student difficulties concerning three-dimensional structures, rotation and reflection. Journal of Chemical Education, 1991, v. 68, n. 6, p. 460-464.

WILEY, S.E. Computer graphics and the development of visual perceptions in engeneering graphics curricula. Engineering Design Graphics Journal, 1990, v. 54, n. 3, p. 39-43.

WILLIAMSON, V. M.; ABRAHAM, M. R. The effects of computer animation on the particulate mental models of college chemistry students, Journal of Research in Science Teaching, 1995, v. 32, p. 521-534.

WU, H.; KRAJCIK, J.S.; SOLOWAY, E. Promoting understanding of chemical representations: students' use of visualization tool in the classroom. Journal of Research in Science Teaching, 2001, v. 38 , n. 7 , p. $821-842$. 


\section{Educational telematics and the teaching of chemistry: considerations about a constructor of molecular objects Abstract}

In this article, we deal with a specific aspect of the representation of particles by means of computer applications and how this relatively new way of representation is inserted in the scientific education of secondary school students. Firstly, we consider some general aspects of the representation in chemistry basing ourselves on the contributions of the research community on the teaching of chemistry. Then, we discuss the representation of particles also getting support from some studies in the area of Science Education so that we can show the contributions of the studies about the use of computer programs for visualization, with emphasis on the distinction between animations and simulations. In the second part, we describe the functionalities of the computer program for the construction of molecular objects that has been developed in our laboratory and their initial use in secondary school classrooms.

Keywords: Structural representation. Simulation. Animation. Science education. Chemistry teaching.

\section{Télématique éducationnelle et l'enseignement de la chimie : considérations sur un constructeur d'objets moléculaires}

Résumé

Cet article traite d'un aspect spécifique de la représentation de particules par l'entremise de logiciels et comment cette relativement nouvelle manière de représentation est insérée dans l'éducation scientifique des étudiants du secondaire. Em premier lieu, nous considérons des aspects généraux de la représentation em chimie en nous appuyant sur les contributions de la communauté de recherche en enseignement de la chimie. Après, nous traitons de représenter de particules, de nouveau en récupérant quelques études du champs de l'Éducation en Sciences, pour ensuite aprésenter les contributions des études sur l'utilisation de logiciels de visualisation, avec emphase sur la distinction entre animations et simulations. Dans la deuxième partie, nous décrivons les fonctionnalités d'un logiciel de construction d'objets molèculaires qui est développé dans notre laboratoire et ses applications dans les salles de classe de l'enseignement secondaire.

Mots clefs : Représentation structurelle. Simulation. Animation. Éducation en sciences. Enseignement de la chimie.

\section{Telemática educacional y enseñanza de química: consideraciones acerca de un constructor de objetos moleculares}

Resumen

En este artículo, tratamos de un aspecto especifico de la representación de partículas por medio de aplicativos computacionales y como esa forma relativamente reciente de representación se insere en la educación científica de alumnos de la enseñanza secundaria. Inicialmente, consideramos algunos aspectos generales de investigación en la enseñanza de quimica. A seguir, tratamos de la representación de las partículas también rescatando algunos estudios en la area de educación en ciencias, para después presentar las contribuciones de los estudios acerca del uso de aplicativos de visualización, con énfasis en la diferenciación entre animaciones y simulaciones. En la segunda parte, descrivimos las funcionalidades de un aplicativo de construcción de objetos moleculares que está siendo desarrollado en nuestro laboratorio y sus primeras aplicaciones en las aulas de la enseñanza secundaria.

Palabras-clave: Representación estructural. Simulación. Animación. Educación en ciencias. Enseñanza de química. 
